Methods Observational study of 64 preterm neonates during their first five days of life with gestational age (GA) $<32$ weeks or very low birth weight of $<1500$ g. Total of 52 patients treated with caffeine and 12 controls without caffeine were included. Sleep-wake behavior was scored in wakefulness (W), active sleep (AS), and quiet sleep (QS) associated with physical and cerebral regeneration. Individual caffeine concentration of every neonate was simulated with a pharmacokinetic model.

Results For increasing caffeine concentration, W increased, AS decreased, and QS was unchanged for GA $>$ or $=28$ weeks. No caffeine effect for GA $<28$ weeks could be demonstrated. Maturational effects could be seen when comparing preterm neonates of GA $>32$ weeks with a birth weight of $<1500 \mathrm{~g}$ with very preterm neonates born of GA < 32: Neonates born $>32$ weeks had a significantly higher amount of $\mathrm{W}$ and lower percentage of AS.

Conclusions Treatment of apnea and bradycardia as well as stabilization of respiration with standard caffeine treatment is not at cost of QS, i.e. time for physical and cerebral regeneration during sleep remains unchanged. There is an increased fraction of W, alertness and most probably also arousability.

Disclosure(s) Nothing to disclose

\section{P25 COMPARISON OF RENAL FUNCTION ESTIMATION METHODS IN CRITICALLY ILL CHILDREN: A PILOT STUDY}

${ }^{1} \mathrm{E}$ Dhont, ${ }^{2} \mathrm{~T}$ Van Der Heggen, ${ }^{1} \mathrm{~A}$ De Jaeger, ${ }^{1} \mathrm{~J}$ Willems, ${ }^{3} \mathrm{~S}$ De Baere, ${ }^{3} \mathrm{~S}$ Croubels, ${ }^{4} \mathrm{~J}$ Vande Walle, ${ }^{5} \mathrm{p}$ De Paepe, ${ }^{1,6} \mathrm{p}$ De Cock*. ${ }^{1}$ Paediatric Intensive Care Unit; ${ }^{2}$ Paediatrics Department, Ghent University Hospital; ${ }^{3}$ Faculty of Veterinary Medicine, Department of Pharmacology, Toxicology and Biochemistry, Ghent University; ${ }^{4}$ Paediatric Nephrology Department, Ghent University Hospital; ${ }^{5}$ Heymans Institute of Pharmacology, Ghent University; ${ }^{6}$ Pharmacy, Ghent University Hospital, Ghent, Belgium

\subsection{6/archdischild-2019-esdppp.63}

Background Accurate assessment of renal function is crucial in intensive care to guide therapy. Both acute kidney injury and augmented renal clearance (ARC) may compromise outcome. Common formulas to estimate glomerular filtration rate (GFR) are unreliable in critically ill adults. ${ }^{1}$ A comparison of a gold standard technique to assess GFR with these formula-based estimations has never been reported in pediatric intensive care (PICU) patients. Our aim was to evaluate the feasibility of measuring plasma iohexol clearance $\left(\mathrm{CL}_{\mathrm{IOHEX}}\right)$ for GFR assessment in critically ill children and to compare $\mathrm{CL}_{\text {IOHEX }}$ with estimated GFR using the modified Schwartz formula $\left(\mathrm{eGFR}_{\text {Schwartz }}\right)$.

Methods A prospective, interventional study was conducted at the PICU of the Ghent University Hospital, Belgium. Critically ill children without chronic kidney disease were included. After injection of a weight-dependent bolus of iohexol, serial blood samples $(n=6)$ were taken over a 6-hours interval. $\mathrm{CL}_{\text {IOHEX }}$ was compared to $\mathrm{eGFR}_{\text {Schwartz }}$ Correlation between both methods was assessed by a Pearson's correlation coefficient (r).Bland-Altman plots were evaluated to assess bias and limits of agreement (LOA). ARC was defined as a GFR exceeding normal values for age plus two standard deviations. Results 40 patients, median age 16 months (range 15 days 13,6 years), $72,5 \%$ males, were included. No adverse effects related to iohexol were observed. Median $\mathrm{CL}_{\mathrm{IOHEX}}$ was 121 $\mathrm{ml} / \mathrm{min} / 1.73 \mathrm{~m}^{2}$ (range: $43-221 \mathrm{ml} / \mathrm{min} / 1.73 \mathrm{~m}^{2}$ ). ARC was present in 20 patients based on $\mathrm{CL}_{\text {IOHEX }}$. Median
eGFR $_{\text {Schwartz }}$ was $81 \mathrm{ml} / \mathrm{min} / 1.73 \mathrm{~m}^{2}$ (range: $31-131 \mathrm{ml} / \mathrm{min} /$ $1.73 \mathrm{~m}^{2}$ ). Only 1 patient was identified with ARC by $\mathrm{eGFR}_{\mathrm{Sch}-}$ wartz. eGFR $_{\text {Schwartz }}$ was systematically lower than $\mathrm{CL}_{\mathrm{IOHEX}}$. There was a good correlation between $\mathrm{CL}_{\mathrm{IOHEX}}$ and $\mathrm{eGFR}_{\mathrm{Sch}}$ wartz $(\mathrm{r}=0,69 ; \mathrm{p}<0,01)$. Bias was $34 \mathrm{ml} / \mathrm{min} / 1.73 \mathrm{~m}^{2}$ with LOA $\left(-24,5 ; 93 \mathrm{ml} / \mathrm{min} / 1.73 \mathrm{~m}^{2}\right)$

Conclusion $\mathrm{CL}_{\mathrm{IOHEX}}$ was safely used to measure true GFR in critically ill children. eGFR Schwartz $_{\text {systematically underestimates }}$ GFR, especially in patients with ARC and seems not reliable in this patient population.

\section{REFERENCE}

1. Baptista JP, Neves M, Rodrigues L, Teixeira L, Pinho J, Pimentel J (2014) Accuracy of the estimation of glomerular filtration rate within a population of critically ill patients. J Nephrol. 27:403-410.

Disclosure(s) Nothing to disclose

\section{P26 PILOT ON HARMONISING DOSING RECOMMENDATION FOR TERM AND PRETERM NEONATES IN THE NETHERLANDS (NEODOSE PROJECT)}

${ }^{1} \mathrm{MA}$ de Hoop-Sommen ${ }^{*},{ }^{2} \mathrm{TM}$ van der Zanden, ${ }^{3,4} \mathrm{~K}$ Allegaert, ${ }^{2,5} \mathrm{RB}$ Flint, ${ }^{2}$ SHP Simons, ${ }^{2,5} \mathrm{SN}$ de Wildt, NeoDose Consortium. ${ }^{1} \mathrm{KNMP}$, Den Haag; ${ }^{2}$ Erasmus MC - Sophia Childrens Hospital, Rotterdam, The Netherlands; ${ }^{3} \mathrm{KU}$ Leuven, Leuven, Belgium; ${ }^{4}$ Erasmus MC Sophia Childrens Hospital, Den Haag; ${ }^{5}$ Radboud University Medical Center, Radboud Institute for Health Sciences, Nijmegen, The Netherlands

\subsection{6/archdischild-2019-esdppp.64}

Background Many drugs are used off-label in term and preterm neonates, and dosing recommendations for many drugs are lacking in the Dutch Paediatric Formulary (DPF). This results in widely varying dosing regimens used across neonatal intensive care units (NICUs) in the Netherlands. The Neodose pilot project aimed to develop best-evidence national dosing recommendations for (pre)term neonates. Because scientific evidence is scarce, a consensus-based approach was used.

Methods A priority drug list, containing the most frequently used drugs for neonates, was drafted. From this list 22 drugs were selected for further research within the Neodose pilot project. The pilot utilized a two-step approach: First, consensus was established with all Dutch NICUs for neonatal dosing recommendations. Local treatment protocols were retrieved, compared and discussed, leading to consensus-based dosing recommendations. Secondly, we aimed to develop best-evidence dosing recommendations for the following five drugs: acyclovir, ganciclovir, ibuprofen, hydrocortisone and dexamethasone.

Results For 21 of 22 drugs, local dosing guidelines differed significantly. Mostly concerning total daily dose, dosing frequency and route of administration. Little or no distinction is made between treatment of preterm and term neonates. Approximately half of the consensus-based dosing recommendations (45\%) differ in some degree from all local protocols. Comparing the consensus-based dosing recommendations with the available evidence, almost half of the consensus doses were adjusted. The grounds on which dosing recommendations were adjusted differed. Acyclovir-dosing adjustment was based on pharmacokinetics. Hydrocortisone-dosing was adjusted due to new insights after the evidence has been put together. For dexamethasone-dosing, the consensus dose was eventually chosen, because every available trial used a different dosing regimen. 